\title{
Some of the methods used to solve complete and incomplete differential equations
}

\section{BASHEER ABD AL-RIDA SADIQ ${ }^{1}$}

AL-Imam AL- Kadhum College for Islamic Science University basheer.abdrida@alkadhum-col.edu.iq

Article History:Received:11 november 2020; Accepted: 27 December 2020; Published online: 05 April 2021

Abstract
This paper studies the methods used to solve complete and in complete differential equations and types of first
order and second order and Exact differential equation to solve integration general in This equation Fur there
more, and the Special cases to find the integration factor use solve those types of equations is use as
well,supported by a relevant variety of examples.
well,supported by a relevant variety of examples.

\section{Types of first-order differential equations and methods of solving them}

1.1 Definitions in differential equations

Differential equation:A Differential Equation is an equation with a function and one or more of its derivatives, which are of shape:

For Example:

$$
f\left(x, y, \ldots \ldots \ldots, \frac{d y}{d x}, \frac{d^{2} y}{d x^{2}} \ldots \ldots\right)
$$

$$
\frac{d x}{d y} z+y d x=u
$$

The differential equation is classified into:

1- Ordinary differential equation:is a differential equation containing derivatives or differentials Ordinary is a containing one or more functions of one independent variable and the derivatives of those functions.

For Example:

$$
y d x+x d y=e^{z}
$$

2-Partial Differential Equations:It is a differential equation containing partial or differential derivatives, as it includes two or more independent variables

For Example:

$$
\frac{\partial x}{\partial y}=z x
$$

3-Linear Differential Equations:equation that is linear For each of the dependent one or more terms, consisting of the derivatives of the variable with respect to one or more independent variables is known as a differential equation.

4-Linear partial differential equation:It is the equation that is linear with respect to the partial derivatives of dependent or more terms an existing function.

Note:

* The rank of the derivative is the order of the highest derivative in the equation

* The differential equation can be converted from one form to another to facilitate its solution

Solving the differential equation:Say about pursue $y=f(x)$ It is a solution to the differential equation if two conditions are met:

$$
\begin{aligned}
& \text { 1. }\left(x, f(x), f^{\prime}(x) \ldots \ldots \ldots \ldots \ldots \ldots, f^{(n)}(x)\right) \in D, \forall x \in I \\
& \text { 2. }\left(x, f(x), f^{\prime}(x) \ldots \ldots \ldots \ldots \ldots \ldots, f^{(n)}(x)\right) \equiv 0, \forall x \in I
\end{aligned}
$$

Where the solutions of the differential equation are divided into:

The general solution to the differential equation

It is the solution that contains optional constants in the differential equation and it is of the form

Example: Solve the general equation:

$$
\varphi\left(x, y, c_{1}, \ldots, c_{n}\right)
$$

$$
\begin{gathered}
y^{\prime \prime}+4 y=0 \\
y=c_{1} \sin 2 x+c_{2} \cos 2 x
\end{gathered}
$$

Where $C_{1}, C_{2}$ Two optional parameters 
The special solution for the differential equation: is the solution that we obtain by giving numerical values for the existing constants for the general solution of the differential equation

Example :special equation solution

$$
\begin{gathered}
y^{\prime \prime}+4 y=0 \\
2 x+\cos 2 x ; c_{1}=c_{2}=1
\end{gathered}
$$

Important note: We can obtain an abnormal solution(anomaly) to the equation, which is the solution that cannot be obtained from the general solution, but rather we get it during the solution of the equation.

Methods for solving differential equations: Contrary to what many think, many differential equations are not yet solvable, but that can be solved by known methods and exact accuracy are few equations relative to the first, and when solving some of the problems that were found all or some of the solutions:

$$
F\left(x, y, y^{\prime}\right)=0
$$

\subsection{First order differential equations solved with respect to the derivative:}

Definition of ( D.E of with separated )

variables : Each form differential equation $\grave{y}=f(x, y)$ It can be reformulated to pool $d x, x$ At the end of it and $d y, x$ The other end is.

Writes in the shape

$$
d y-F(x, y) d x=0
$$

This equation is called separate variables if $N A$ certified on $x$ and $M$ depends on $y$ It can be written in general:

Integration can be done directly:

$$
M(x) d x+N(y) d y=0
$$

$$
\int M(x) d x+\int N(y) d y=c
$$

By conducting integration, we find that the general solution is

$$
M_{1}(X)+N_{1}(y)=c
$$

Example:

$$
\left(1+3 x^{2}\right) d x+2 y d y=0
$$

They have separate variables General integration it : $x+x^{3}+y^{2}=c$

There are a large number of equations that can be converted separately Changing some simple operations and we will discuss several cases that can be converted to this shape:

\section{1-Equation for shape *}

$$
f_{2}(y) d x+g_{1}(d x) d y=0
$$

Where we return it to separate variables by dividing by $f(x), g(y)$

It becomes separate variables of the shape:

$$
\frac{d x}{f(x)}+\frac{d y}{g(y)}=0
$$

\section{2-Equation for shape **}

$$
M_{1}(X) \cdot M_{2}(y) d x+N_{1}(x) \cdot N_{2}(y) d y=0
$$

Where we return it to the same separate variables by dividing its ends by $M_{2}(y) \cdot N_{1}(x)$

To become:

\section{3-Equation for shape $* * *$}

$$
\frac{M_{1}(x)}{N_{1}(x)} d x+\frac{N_{2}(y)}{M_{2}(y)} d y=0
$$

$$
\grave{y}=f(y+a x+b)
$$

It becomes separate and that changes in the dependent by a relationship of form:

$$
z=y+a x+b
$$

With compensation we find thatỳ $+a=$ źWith compensation it becomes a form:

$$
z^{\prime}=F_{1}(z)
$$

You write as:

$$
d x=\frac{d z}{f_{1(z)}}
$$

Its general solution: 
Returning to the previous equation, we find that

$$
x-c=f(z)
$$

$$
x-c=F(y+a x+b)
$$

Note that any root of the equation $F(z)=0$ It may be a solution to the equation

\section{4-Equation for shape****}

in which $g$, $f$ Continued follower

$$
y^{\prime}=f(x) g(y)
$$

As this equation writing for a shape:

$$
\begin{gathered}
\frac{d y}{d x}=f(x) g(y) \\
\text { Or } \\
\frac{d y}{g(y)}=f(x) d x ; \forall g(y) \neq 0
\end{gathered}
$$

They become separate mutants

if it was $g(a)=0$ Was the followerFixed $y \equiv a$ A solution to the differential equation

\subsection{Exact differential equation}

So be it $M(x, y) N(x, y)$ Followers are known and continuous Since they are not lacking in one, we call the

$$
\text { 1) } M(x, y) d x+N(x, y) d y=0
$$
differential equation written as shape :

With a full differential equation if the left side is a complete Differential of a follower like $U=(x, y)$ If not, then a differential equation is not complete.

Theorem: Suppose that the differential equation is complete then there is a Follower $U(x, y)$ So that its total differential is identical to the lefi side:

$$
d u=\frac{\partial U}{\partial x} d x+\frac{\partial U}{\partial y} d y \equiv M(x, y) d x+N(x, y) d y=0
$$

where integration general in This equation is given in the form:

$$
U(x, y)=c
$$

Example: $\left(3 x^{2} y+8 x y^{2}\right) d x+\left(x^{3}+8 x^{2} y+12 y^{2}\right) d y=0$ Where it can be written as shape :

Then shape

$$
\left(3 x^{2} y d x+x^{3} d y\right)+\left(8 x y^{2} d x+8 x^{2} y d y\right)+12 y^{2} d y=0
$$

Where he Integration group complete She writes as shape

$$
d\left(x^{3} y\right)+d\left(4 x^{2} y^{2}\right)+d\left(4 y^{3}\right)=0
$$

$$
\begin{gathered}
d\left(x^{3} y+4 x^{2} y^{2}+4 y^{3}\right)=0 \\
U(x, y)=x^{3} y+4 x^{2} y^{2}+4 y^{3}=c
\end{gathered}
$$

Definition: We call a peaceful linear differential equation of the first order, each equation of shape or convertible of form ỳ $=a(x) y+b(x)$

Where two continued on the open space $I$

Theorem: Let $I$ it be an open and incomplete field From $\mathrm{R}$ and So be it $a a n d b$ Continuing followers On the field and take the values in R So be it $x \rightarrow A(x)$ Original follower of the follower $x \rightarrow a(x)$ on $I$ and $x \rightarrow B(x)$ set of

$$
y^{\prime}=a(x) y+b(x)
$$
solutions differential equation

she $S=\{\varnothing: \wedge \in C\}$ Where

Evidence we've got

$$
\emptyset: I \rightarrow C, \emptyset(x)=\Lambda \cdot e^{A(x)} \cdot B(x)
$$

$$
\begin{gathered}
\forall x \in I, \emptyset^{\prime}(x)=\lambda A^{\prime}(x) \cdot e^{A(x)}+A^{\prime}(x) \cdot e^{A(x)} \cdot B(x)+e^{A(x)} \cdot B^{\prime}(x) \\
=\lambda \mathrm{a}(\mathrm{x}) e^{A(x)}+a(x) \cdot e^{A(x)} \cdot B(x)+e^{A(x)} \cdot b^{\prime}(x) \cdot e^{-A(x)} \\
=a(x) \cdot \emptyset(x)+b(x)
\end{gathered}
$$

From it is a solution to the equation and vice correct

Integration factors and methods of finding them

\section{Methodology}

\subsection{Intergrating factor $(x, y)$}

We use the complement factor to convert an incomplete equation to an exact, since it is one way to solve an incomplete differential equation.

Definition: We say that the Follower $\mu(x, y)$ Factor if the equation after multiplying becomes a completely differential equation.

where the integration factor $\mu$ Must achieve the following Matching:

$$
\frac{\partial(\mu M)}{\partial y}=\frac{\partial(\mu N)}{\partial x}
$$


With the necessary derivation procedure, we find that:

After the arrangement, we find that:

$$
M \frac{\partial \mu}{\partial y}+\mu \frac{\partial M}{\partial y}=N \frac{\partial \mu}{\partial x}+\mu \frac{\partial N}{\partial x}
$$

$$
N \frac{\partial \mu}{\partial x}-M \frac{\partial \mu}{\partial y}=\mu\left(\frac{\partial M}{\partial y}-\frac{\partial N}{\partial x}\right)
$$

Partial differential equation of the first order relative to the Follower the unknown $\mu$ It is called the Lagrange equation And we can find a special solution for it By imposing and renewing

Integration Process The Follower of $(x)$

The requirement for an integration process $\mu=\mu(x)$ should investigate $\frac{\partial \mu}{\partial y}=0$ And to achieve $\frac{\partial \mu}{\partial x}=\frac{d \mu}{d x}$ And the previous equation becomes on shape:

$$
\frac{d \mu}{\mu}=\frac{\frac{\partial M}{\partial y}-\frac{\partial N}{\partial x}}{N} \quad, N(x, y) \neq 0
$$

Here we note that if the right side is affiliated with $x$ Only it can be easily integrated and it can be Designation:

Including that:

$$
\frac{\frac{\partial M}{\partial y}-\frac{\partial N}{\partial x}}{N}=f(x)
$$

By the integration of the two parties, we find that:

$$
\frac{d \mu}{\mu}=f(x) d x
$$

$$
\mu=c \cdot e^{\int f(x) d x}=c \cdot \mu(x)
$$

As for if the right side is not The Follower with of $x$ Only the equation is not integral

Example: $\left(x^{2}+x-y^{2}\right) d x-x y d y=0$

Not complete, because:

$$
\frac{\partial M}{\partial y}=-2 y, \frac{\partial N}{\partial x}=-y, \frac{\partial M}{\partial y} \neq \frac{\partial N}{\partial x}
$$

And let's try to find a complement to this equation of form $\mu=\mu(x)$ And by the condition we find that:

That is, the condition is fulfilled

$$
\frac{\frac{\partial M}{\partial y}-\frac{\partial N}{\partial x}}{-x y}=\frac{1}{x}=f(x)
$$

In integral, we find that:

After multiplying by the integral factor:

$$
u(x)=e^{\left(\int f(x) d x\right)}=e^{\int \frac{d x}{x}}=e^{\ln (x)}=x
$$

$$
\left(x^{3}+x^{2}-x y^{2}\right) d x-x^{2} y d y=0
$$

Using the previous relationship where $x_{0}=0, y_{0}=0$ The general integration of the equation can be obtained By adding the limits by writing the equation as shape:

$$
\begin{gathered}
x^{3} d x+x^{2} d x-\left(x y^{2} d x+x^{2} y d y\right)=0 \\
\rightarrow 3 d\left(\frac{x^{4}}{4}+\frac{x^{3}}{3}\right)-\frac{1}{2} d\left(x^{2} y^{2}\right)=0
\end{gathered}
$$

And the general solution.

$$
3 x^{4}+4 x^{3}-6 x^{2} y^{2}=12 c_{1}=c
$$

\section{Integration Process The Follower of $(y)$}

The requirement for an integration process the Follower of $y$ Just any $\mu=\mu(y)$

It is to be

Where the equation takes the form:

$$
\frac{\partial \mu}{\partial y}=\frac{d \mu}{d y}, \frac{\partial \mu}{\partial x}=0
$$

$$
\frac{\partial \mu}{\partial y}=\frac{\frac{d \mu}{d y}-\frac{\partial \mu}{\partial x}}{-M}=g(y) \quad ; M(x, y) \neq 0
$$

Where if the left continued the Follower of $y$ The equation can be solved And with the complementarity of the previous relationship, we find that: 


\section{Example:}

$$
u(y)=e^{\int f(x) d x} ; c=1
$$

The solution: This equation is not complete:

$$
\left(2 x y^{4} e^{y}+2 x y^{3}+y\right) d x+\left(x^{2} y^{4} e^{y}-x^{2} y^{3}-3 x\right) d y=0
$$

$$
\begin{gathered}
\frac{\partial M}{\partial y}=8 x y^{3} e^{y}+2 x y^{4} e^{y}+6 x y^{2}+1, \frac{\partial N}{\partial x}=2 x y^{4} e^{y}-2 x y^{2}-3 \\
\frac{\partial M}{\partial y} \neq \frac{\partial N}{\partial x}
\end{gathered}
$$

9Let's look for a complement to the formula from the figure $\mu=\mu(y)$ We find that:

From it:

$$
\frac{\frac{\partial M}{\partial y}-\frac{\partial N}{\partial x}}{-M}=\frac{4\left(2 x y^{3} e^{y}+2 x y^{2}+1\right)}{y\left(2 x y^{3} e^{y}+2 x y^{2}+1\right)}=-\frac{4}{y}=g(y)
$$

$$
u(y)=e^{\int \mathrm{g}(\mathrm{y}) \mathrm{dy}}=\frac{1}{y^{4}}
$$

Let's multiply both sides of the given equation by the complement factor $\frac{1}{y^{4}}$ Get the full differential equation next:

From it we find that:

$$
\left(2 x e^{y}+\frac{2 x}{y}+\frac{1}{y^{3}}\right) d x+\left(x^{2} y-\frac{x^{2}}{y}-\frac{3 x}{y^{4}}\right) d y=0
$$

The general complementarity of the differential equation is:

$$
\mu(x, y)=\int_{0}^{x}\left(2 x e^{y}+\frac{2 x}{y}+\frac{1}{y^{3}}\right) d x+0=c
$$

$$
x^{2} c^{y}+\frac{x^{2}}{y}+\frac{x}{y^{3}}=c
$$

Where the exact equation can be converted to the sum of the exact equation differentials.

$$
\left(2 x e^{y} d x+x^{2} e^{2} d y\right)+\left(\frac{2 x d x}{y}-\frac{x^{2}}{y^{2}} d y\right)+\left(\frac{1}{y^{3}} d x-\frac{3 x}{y^{4}} d y\right)=0
$$

Thus, he writes in the as shape of one complete differentiation:

In complete we find that the general integration is:

$$
d\left(x^{2} e^{y}+\frac{x^{2}}{y}+\frac{x}{y^{3}}\right)=0
$$

$$
x^{2} e^{y}+\frac{x^{2}}{y}+\frac{x}{y^{3}}=c
$$

\subsection{Special cases to find the integration factor:}

\section{Method 1:}

If we can put the differential equation as a sum of two differential equations from the figure:

$$
M_{1}(x, y) d x+N_{1}(x, y)+M_{2}(x, y) d x+N_{2}(x, y)=0
$$

Sometimes the complement factor of this equation can be found after the complement factors of the two equations are known

$$
\begin{aligned}
& M_{1}(x, y) d x+N_{1}(x, y)=0 \\
& M_{2}(x, y) d x+N_{2}(x, y)=0
\end{aligned}
$$

Suppose $U_{1}$ The complementary factor to the first equation $U_{1}(x, y)=C$ and that Integration factor of the second equation $U_{2}(x, y)$ Where there is an integral factor for the main equation If the following condition is met So that it is:

Example: Solving a differential equation:

$$
u_{1} f_{1}\left(U_{1}\right)=u_{2} f_{2}\left(U_{2}\right)
$$

Note that the differential equation:

$$
x^{2} y^{3}(4 y d x+5 x d y)+8(y d x+x d y)=0
$$

Acceptance of an integrator:

$$
8(x d y+y d x)=0
$$

$$
u_{1}=\frac{1}{x y}
$$


Its general solution:

The differential equation:

$$
U_{1}(x, y)=x y=c
$$

$$
x^{2} y^{3}(4 y d x+5 x d y)=0
$$

Accept an integration factor:

And its general solution:

$$
u_{2}=\frac{1}{x^{3} y^{4}}
$$

$$
U_{2}(x, y)=x^{4} y^{5}=c_{2}
$$

Let's look for the followers $f_{1}, f_{2}$ So that it is:

as :

$$
\frac{1}{x y} f_{1}(x y)=\frac{1}{x^{3} y^{4}} f_{2}\left(x^{4} y^{5}\right)
$$

From it must be achieved:

$$
x^{n-1} \cdot y^{n-1}=x^{4 m-3} \cdot y^{5 m-4}
$$

From them we find

$$
n-1=4 m-3, n-1=5 m-4
$$

$$
U=\frac{1}{x y}(x y) U=\frac{1}{x y}(x y)^{2}=x y
$$

It is an integral factor for the differential equation After multiplying by the complement factor, the equation becomes from the form:

or:

$$
d\left(x^{3} y^{5} d x+5 x^{4} y^{4} d y\right)+18 x y(y d x+x d y)=0
$$

And its overall integration:

$$
d\left(x^{4} y^{5}+4 x^{2} y^{2}\right)=0
$$

$$
x^{4} y^{5}+4 x^{2} y^{2}=c
$$

\section{Method 2:}

If the equation can be written on the figure:

Suppose $\quad z=f(x, y)$

$$
d f(x, y)+M(x, y) d x+N(x, y) d y=0
$$

If possible, assign an integral factor to the differential equation:

$$
M(x, y) d x+N(x, y) d y=0
$$

About $z$ Only this factor was a complement to the original equation.

\section{Method 3:}

In some cases it is possible to move from the variables $x, y$ Into new transformers $u, v$ where $u=g(x, y)$ and $v=\emptyset(x, y)$ This is to find a complement to the equation

Example:

Solve the following differential equation:

solution:

$$
y^{2} d x+(x y+\operatorname{tg} x y) d y=0
$$

$$
\frac{\partial M}{\partial y}=2 y, \frac{\partial N}{\partial x}=y+y\left(1+\tan ^{2} x y\right), \frac{\partial M}{\partial y} \neq \frac{\partial N}{\partial x}
$$

assume that: $x y=\mu$ or $x=\frac{u}{y} \mathrm{We}$ find that:

$$
d x=\frac{y d u-u d y}{y^{2}}
$$

With compensation:

$$
y d u+\text { tanudy }=0
$$

It is of the shape

$$
M(u, y) d u+N(u, y) d y=0
$$

look for the integral factor of the shape $\mu=\mu(x)$ :

$$
\begin{gathered}
\frac{\partial M}{\partial y}=1, \frac{\partial N}{\partial u}=1+\tan ^{2} u \\
\frac{\partial M}{\frac{\partial y}{2}-\frac{\partial N}{\partial u}}=\frac{1-1-\tan ^{2}}{\tan u}=-\tan u=f(u)
\end{gathered}
$$


And get the integration factor:

$$
u=e^{\int \mathrm{f}(\mathrm{x}) \mathrm{dx}}=\cos \cos u
$$

Multiply both sides of the equation by the integral factor, and we find:

$$
y \cos \cos u d u+\sin
$$

The general integration is:

$$
y \cos \cos u d u+\sin \sin u d y=d(y \sin \sin u)=0
$$

Referring to the Variables we find:

$$
\begin{gathered}
y \sin \sin u=c \\
y \sin \sin (x \cdot y)=c
\end{gathered}
$$

\section{References}

A. Kudeshia, A.K. Jagannatham, (2014). "Optimal viterbi based total variation sequence detection TVSD for robust image/video decoding in wireless sensor networks", IEEE Signal Processing Letters, vol. 21, pp. 722 7261. Mohamed Adel Swaidan ,Mathematical analysis ,Damascus University Press.,the third part 1982.

Dr. Katour Imran Quba, Analysis, Part Three, Higher Institute of Applied Sciences and Technology 2002

Dr. Ziyad Al-Amir, differential equations, Directorate of books for university publications.1992

Mohammed, A.H. , Sadiq, B.A. , Hassan,A.M. "Solving New Type ofLinear Equations by Using New Transformation”EUROPEA ACADEMIC RESEARCH Vol. IV, Issue 8/ November 2016

James C. Robinson, “An Introduction to Ordinary Differential Equations”, Cambridge University Press, New York, 2004.

Ismaeel Bukfa, Aish alhindawa,: Differential Equations, solutions and applications, Sconce and Technology University, Yemen Republic: 2008.

Bikheet Naqee' Almatrafi, Abdullah Abdullah Musa: Differential Equations, theory and application, Altaif University, Scientific Publication Administration: 2012.

Hassan Mustafa Alouwaidhi, Abdulwahab Abbas Rajab, Sana' Zarra': Differential Equations, part 1, Alazhar University, Publication press: 2010.

Jalal Alhaj Abid: Ordinary and Partial Differential Equations, Winter Library: 2008.

Ramadhan Mohammed Jaheema, Ahmed Abdulali Hab Alreeh: Calculus/Part2/3rd edition/ Alkitab Press, Alhareer united/2002

Alhayani B., H. Ilhan.(2020). "Image transmission over decode and forward based cooperative wireless multimedia sensor networks for Rayleigh fading channels in medical internet of things (MIoT) for remote health-care and health communication monitoring," Journal of Medical Imaging And Health Informatics, vol. 10, pp. 160-168.

/10.1108/EC-02-2020-0107.

B. .Alhayani, Milind Rane. (2014). "face recognition system by image processing" International journal of electronics and communication engineering \& technology (IJCIET), vol.5, no.5, pp. 80-90.

T. Ma, M. Hempel, K. Hua, D. Peng and H. Sharif,( 2010,) "A novel cooperative image transmission scheme in Wireless Sensor Networks," IEEE Local Computer Network Conference, Denver, CO, pp. 240-243.

Mahajan, H.B., Badarla, A. \& Junnarkar, A.A. (2020). CL-IoT: cross-layer Internet of Things protocol for intelligent manufacturing of smart farming. J Ambient Intell Human Comput. https://doi.org/10.1007/s12652020-02502-0.

Mahajan, H.B., \& Badarla, A. (2018). Application of Internet of Things for Smart Precision Farming: Solutions and Challenges. International Journal of Advanced Science and Technology, Vol. Dec. 2018, PP. 37-45. Mahajan, H.B., \& Badarla, A. (2019). Experimental Analysis of Recent Clustering Algorithms for Wireless Sensor Network: Application of IoT based Smart Precision Farming. Jour of Adv Research in Dynamical \& Control Systems, Vol. 11, No. 9. 10.5373/JARDCS/V11I9/20193162. 
Mahajan, H.B., \& Badarla, A. (2020). Detecting HTTP Vulnerabilities in IoT-based Precision Farming Connected with Cloud Environment using Artificial Intelligence. International Journal of Advanced Science and Technology, Vol. 29, No. 3, pp. 214 - 226.

Mikhail, A., Kamil, I. A., \& Mahajan, H. (2017). Increasing SCADA System Availability by Fault Tolerance Techniques. 2017 International Conference on Computing, Communication, Control and Automation (ICCUBEA). doi:10.1109/iccubea.2017.8463911

Mikhail, A., Kareem, H. H., \& Mahajan, H. (2017). Fault Tolerance to Balance for Messaging Layers in Communication Society. 2017 International Conference on Computing, Communication, Control and Automation (ICCUBEA). doi:10.1109/iccubea.2017.8463871 